\title{
Suplementación con Omega-3 para prevención de parto prematuro recurrente: revisión sistemática y metaanálisis (1)
}

\author{
Saccone G, Berghella V. Omega-3 supplementation to prevent recurrent preterm birth: \\ a systematic review and metaanalysis of randomized controlled trials. Am J Obstet Gy- \\ necol. 2015;213(2):135-40.
}

Análisis crítico: Camila Fernández N, Jorge Carvajal C, PhD.

Unidad de Medicina Materno-Fetal, División de Obstetricia y Ginecología, Facultad de Medicina, Pontificia Universidad Católica de Chile.

\section{RESUMEN (1)}

Objetivo: Evaluar la eficacia de los suplementos de Omega-3 para la prevención del parto prematuro $(\mathrm{PP})$ recurrente en gestaciones únicas asintomáticas con PP previo. Método: Se realizó una búsqueda con los términos: aceite de pescado, ácidos grasos poliinsaturados de cadena larga, embarazo y Omega-3, en MEDLINE, OVID, Scopus, ClinicalTrials.gov, el Registro prospectivo Internacional de Revisiones Sistemáticas PROSPERO, EMBASE y el Registro Cochrane de Ensayos Controlados desde el inicio de cada base de datos a diciembre de 2014, sin límite por lenguaje. Además se examinaron las listas de referencias de todos los artículos identificados para identificar los estudios que no fueron capturados por las búsquedas electrónicas. Se realizó un metaanálisis de ensayos controlados aleatorizados de gestaciones únicas asintomáticas con PP previo que fueron asignados al azar a suplementación profiláctica con Omega-3 versus control (placebo o ningún tratamiento). El resultado primario fue predefinido como $\mathrm{PP}<37$ semanas de gestación. Los resultados agrupados se informaron como riesgo relativo (RR) con un intervalo de confianza del 95\% (IC 95\%). El protocolo de esta revisión se ha registrado en el PROSPERO (número de registro: CRD42015016371). Se analizaron dos ensayos controlados aleatorios que incluyeron 1080 mujeres. Resultados: La media de edad gestacional en el momento de la aleatorización fue de aproximadamente 134 días en ambos grupos (diferencia, 0,01 días; IC 95\%-0,13 a 0,14). Las mujeres que recibieron ácidos grasos omega-3 tenían tasas similares de PP <37 semanas de gestación (34,5\% vs $39,8 \%$; RR 0,81 ; IC $95 \% 0,59-1,12)$ y PP <34 semanas de gestación (12,0\% vs $15,4 \%$; RR 0,62; IC 95\% 0,26-1,46) en comparación con los sujetos control. El grupo suplementado con Omega-3 tenía una latencia (diferencia media de 2,10 días; IC $95 \% 1,98-2,22)$ significativamente más larga y un mayor peso al nacer (diferencia media 102,52 g, IC $95 \%$ 20,09-184,95), en comparación con los sujetos control. Los otros resultados secundarios (que incluyeron la edad gestacional al momento del parto, PP espontáneo <37 y 34 semanas de gestación, ingreso a una unidad de cuidados intensivos neonatales, hemorragia intraventricular, enterocolitis necrotizante, sepsis y muerte perinatal) fueron similares. Conclusión: La suplementación con Omega-3 durante el embarazo no reduce la recurrencia de PP en gestaciones únicas asintomáticas con antecedente de PP previo. Los beneficios en una latencia más larga y mayor peso al nacer pueden merecer un mayor estudio.

\section{ANÁLISIS DE LA INVESTIGACIÓN}

\section{A. Relevancia clínica de la investigación}

El parto prematuro (PP) representa el $70-75 \%$ de la mortalidad perinatal y es responsable del $50 \%$ de las discapacidades del desarrollo neurológico a largo plazo $(2,3)$. Su incidencia en Chile ha aumentado llegando a cifras del 7,2\% al $2011(4,5)$, destacando dentro de sus múltiples factores de riesgo el antecedente de un parto prematuro previo. Existen múltiples mecanismos involucrados en la 
etiopatogenia del parto prematuro; dentro de estos, se ha descrito un aumento en la concentración de prostaglandinas en la circulación materna previo al inicio del trabajo de parto prematuro, del mismo modo la administración exógena de prostaglandinas induce dilatación cervical y contracciones uterinas $(6,7)$. En este contexto, se ha propuesto la suplementación de ácidos grasos Omega 3 como una alternativa preventiva del parto prematuro, puesto que estos disminuirían la síntesis de prostaglandinas (8). Otros grupos han postulado que la suplementación con ácidos grasos Omega 3 podría mejorar la tasa de parto prematuro al mejorar la placentación (9). Sin embargo, estudios clínicos randomizados muestran resultados contradictorios sobre la utilidad de la suplementación materna con ácidos grasos Omega 3 para reducir la tasa de parto prematuro. Este estudio ofrece un meta análisis que evalúa la eficacia de la suplementación de Omega-3 durante el embarazo para reducir la recurrencia de parto prematuro en gestantes asintomáticas con antecedente de parto pre término previo.

\section{B. El estudio (1)}

Diseño: Revisión sistemática y metaanálisis. Pacientes: Se buscó en las bases de datos MEDLINE, OVID, Scopus, ClinicalTrials.gov, PROSPE$\mathrm{RO}$, Embase, Science direct y Cochrane, todos los estudios clínicos ramdomizados (ECR) publicados hasta diciembre de 2014, que evaluaran el uso de ácidos grasos poliinsaturados durante el embarazo. Los estudios fueron analizados por dos investigadores independientes, los desacuerdos fueron resueltos por consenso o por un tercer investigador. Los criterios de inclusión fueron ECR que incluyeran mujeres con embarazos únicos asintomáticas con antecedente de parto prematuro, ramdomizadas a tratamiento profiláctico con Omega 3 o control (placebo o no tratamiento). Se evaluaron 7850 estudios, tras seleccionar aquellos ECR quedaron 29, de los que fueron revisados los artículos completos y se descartaron 27 por no incluir pacientes con antecedente de PP. Finalmente, se seleccionaron dos artículos para analizar, con un total de 1080 embarazos únicos con el antecedente de al menos un PP previo; 544 pacientes $(50,4 \%)$ fueron asignadas al grupo tratamiento, mientras que 540 (49,6\%) lo fueron al grupo control. Todos los análisis fueron realizados por intención de tratar. Intervención: Suplementación diaria con ácido docosahexaenoico (800-900mg) y eicosapentaenoico (1200-1300 mg), iniciado en promedio a los 134 días de gestación (19 semanas). Resultado primario: Incidencia parto pretérmino <37 semanas edad gestacional (EG). Resultado secundario: EG al momento del parto, intervalo desde la randomización hasta el parto (latencia), PP <34 sem EG, PP espontáneo <34 sem y $<37$ sem y outcome neonatal incluyendo: peso de nacimiento, admisión a la unidad de cuidado intensivo neonatal, síndrome de distrés respiratorio neonatal, displasia broncopulmonar, hemorragia intraventricular, enterocolitis necrotizante, sepsis neonatal y muerte perinatal. Resultados: Las mujeres que recibieron suplementación con Omega-3 presentaron similares tasas de parto prematuro <37 sem EG (34,5\% vs 39,8\%; RR 0,81; IC 95\%: 0,59$1,12)$, y <34 sem EG (12,0\% vs $15,4 \%$; RR 0,62 ; IC 95\%: 0,26-1,46) en comparación con mujeres en el grupo control. El grupo tratado presentó significativamente mayor latencia (2,10 días; IC 95\%: 1.98-2.22) y mayor peso de nacimiento (diferencia promedio: 102,52 g; IC 95\%: 20,09-184,95) comparado con grupo control. El resto de los resultados secundarios fue similar en ambos grupos.

\section{Análisis crítico}

Validez interna: Se utilizaron las bases de datos más importantes disponibles y no se limitó la búsqueda a un idioma. Analizaron la bibliografía de cada uno de los estudios seleccionados para identificar los estudios que no fueron capturados por las búsquedas electrónicas. Se contactaron con los autores de los estudios incluidos obteniendo data no publicada adicional, todo lo anterior hace improbable que estudios importantes y relevantes se hayan pasado por alto. El estudio tiene una descripción adecuada de criterios de inclusión y exclusión. Los criterios de inclusión permiten obtener el grupo de estudio definido: mujeres cursando embarazo único con antecedentes de parto prematuro. Se especifica claramente cómo los autores seleccionaron y/o descartaron los estudios. Además, los dos estudios incluidos fueron estudios clínicos randomizados, de suplementación con Omega 3 versus placebo. Los grupos estudiados eran similares en ambos estudios, al igual que el tipo de ácido graso poliinsaturado y la dosis. Se evaluó el riesgo de sesgo de cada estudio según los criterios descritos en el manual Cochrane para revisiones sistemáticas, esta incluye evaluación de secuencia de generación de randomización, ocultamiento de secuencia, ciego de participantes y personal a cargo, ciego de resultados, seguimiento incompleto, reporte selectivo de eventos de interés y otras fuentes de sesgo. Ambos estudios fueron clasificados como de bajo riesgo de sesgo. Los autores estimaron la heterogeneidad utilizando los test $\mathrm{I}^{2}$ y $\mathrm{Chi}^{2}$, en caso de heterogeneidad estadísticamente significativa se utilizó un modelo de efectos aleatorios, de lo contrario un modelo de efectos fijos.

Conclusiones: La revisión sistemática y metaanálisis cumple con los criterios para establecer que se ha evitado el sesgo (pregunta clara y precia, y criterios de búsqueda, inclusión y exclusión bien definidos). Los estudios incluidos en el análisis 
tienen un adecuado diseño (ECR) para establecer causalidad, con una adecuada valoración del riesgo de sesgo. Sin embargo, uno de los dos estudios analiza varios resultados clínicos, y combina los factores de riesgo de recurrencia, es decir, no fue específicamente diseñado para evaluar la recurrencia de parto prematuro. El segundo estudio fue específicamente diseñado para estudiar prevención de recurrencia de parto prematuro. El resultado del metaanálisis de estos dos estudios no apoya el uso de suplementación con Omega-3 durante el embarazo para prevención de recurrencia de parto prematuro. Estos resultados desaconsejan el uso de suplementación con omega-3 durante el embarazo como un modo rutinario para prevención secundaria del parto prematuro. Sin embargo, muestran la falta de evidencia final para adoptar una decisión en este tema, haciendo necesario efectuar estudio clínicos prospectivos, randomizados, que apunten específicamente a responder la pregunta de la utilidad de suplementación con Omega-3 para prevención secundaria, pero también primaria. Es importante notar que en los dos estudios incluidos, la suplementación se inicia desde las 16 semanas en adelante. Es posible que si el efecto de suplementación con Omega-3 se relacione con mejorar la placentación, la suplementación deba ser iniciada a menor edad gestacional.

\section{REFERENCIAS}

1. Saccone G, Berghella V. Omega-3 supplementation to prevent recurrent preterm birth: a systematic review and metaanalysis of randomized controlled trials. Am J ObstetGynecol 2015;213(2):135-40.

2. Goldenberg R, Culhane J, Romero R. Epidemiology and causes of preterm birth. Lancet 2008;371(9606):75-84.

3. Mathews TJ, Menacker F, MacDorman MF. Infant mortality statistics from the 2002 period: linked birth/ infant death data set. Natl Vital Stat Rep 2004;53:129.

4. Nacido Demasiado Pronto: Informe de Acción Global sobre Nacimientos Prematuros. www.who.int/pmnch/ media/news/2012/preterm_birth_report/es/index.html

5. Estadística Nacional. Nacidos vivos inscritos según semanas de gestación, por región y comuna. Chile, 2011. DEIS. http://www.deis.cl/?page_id=3295

6. Olsen SF, Hansen HS, Sorensen TI, et al. Intake of marine fat, rich in (n-3)-polyunsaturated fatty acids may increase birthweight by prolonging gestation. Lancet 1986;2:367-9.

7. Olsen SF, Secher NJ, Tabor A, Weber T, Walker JJ, Gluud C. Randomised clinical trials of fish oil supplementation in high risk pregnancies: Fish Oil Trials in pregnancy (FOTIP). BJOG 2000;107:382-95.

8. Harper M, Thom E, Klebanoff MA, et al. Omega-3 fatty acid supplementation to prevent recurrent preterm birth: a randomized controlled trial. Obstet Gynecol 2010;115:234-42.

9. Carvajal JA. Docosahexaenoic acid supplementation early in pregnancy may prevent deep placentation disorders. Biomed Res Int 2014;2014:526895. 